\title{
A Multimodal and Multisensorial Pre-Operative Planning Environment for Total Hip Replacement
}

\author{
D Testi ${ }^{1}$, C Zannoni ${ }^{2}$, M Petrone ${ }^{2}$, G J Clapworthy ${ }^{3}$, D Nieberg ${ }^{4}$, \\ N G Tsagarakis ${ }^{5}, D_{\text {G Caldwell }}^{5}$, M Viceconti $^{1}$ \\ ${ }^{1}$ Laboratorio di Tecnologia Medica, Istituti Ortopedici Rizzoli, Bologna, Italy \\ ${ }^{2}$ Supercomputing Centre, CINECA, Casalecchio di Reno, Bologna, Italy \\ ${ }^{3}$ Department of Computing \& Information Systems, University of Luton, UK \\ ${ }^{4}$ Department of Speech, Music and Hearing, KTH, Sweden \\ ${ }^{5}$ School of Computer Science and Engineering, University of Salford, UK \\ emailaddresses:testi@tecno.ior.it,c.zannoni@cineca.it,m.petrone@cineca.it, \\ gordon.clapworthy@luton.ac.uk,neiberg@speech.kth.se,n.tsagarakis@salford.ac.uk, \\ viceconti@tecno.ior.it
}

\begin{abstract}
This paper describes a new environment for the pre-operative planning of total hip replacement. The system is based on a multimodal/multisensorial interface, which includes advanced software visualisation and evaluation modules for the planning and state-of-the-art technologies for immersive interface (stereoscopic display, different six degrees of freedom tracking technologies, speech recognition, and haptic feedbacks). This paper is focused on the final clinical application description. More specific visualisation-related modules are described in other related papers.
\end{abstract}

\section{Introduction}

Improvements in computer science have, in recent years, allowed the consideration of specialised Virtual Reality (VR) approaches to medical applications. In particular, the use of immersive environments have become of great interest in the training of clinical personnel [1]. VR techniques have been already used in neuro [2], craniofacial [3], and orthopaedic surgery [4].

Pre-operative planning is a fundamental phase in total hip replacement (THR) surgery [5] and the advantage of using a $3 \mathrm{D}$ environment for it has been demonstrated [6]. Different systems for preoperative planning of THR have been developed in recent years $[4,7,8]$. Although it is more intuitive to grasp and examine a virtual object within an immersive system, the navigation is mostly achieved by using a two- degrees-of-freedom mouse and a flat screen for pseudo-3D interaction.

At present, no pre-operative planner includes soft tissue modelling, and usually, when planning the placement of the cup and stem components, the femur and the ileum are visualised separately. Another limitation of these systems is in the relative lack of biomechanical evaluation of the achieved position.

From this background, the Multisense project started with the aim of combining different sensorial devices (haptics, speech, stereoscopic visualisation, and six- degrees-of-freedom tracking) in a unique VR environment for pre-operative planning of THR. While the demonstrator of this multisensorial integration is focused on a specific clinical application, the effectiveness of the approach, after validation, will also be of great relevance to many other orthopaedic and medical applications.

\section{Total Hip Replacement}

This section describes THR surgery for cementless components so that the various aspects of the system developed can be put in context.

In THR, the surgeon seeks to access the proximal part of femur and the cavity in the pelvis in which the head of the femur sits - the acetebulum. To do this, an incision is made in the skin of the thigh, and metal retractors are put in place at the lip of this incision to keep it open. Each prosthesis manufacturer will have a recommended procedure for fitting the prosthesis and this will include defining the position of the initial incision. However, in Multisense, we need to take 
account of special cases where the patient presents particular problems to the surgeon, perhaps because of physical abnormality. The surgical planner is even more valuable in such cases than in a standard THR, which is why it is important that a patient-specific anatomical model is available for the planning.

The surgeon then attempts to move past the various muscles that are blocking access to the site of the surgery. This is normally performed by retracting the muscles individually, with assistants using retractors to hold back muscles already displaced. In cases where retraction of a muscle is not suitable, it may be cut to allow the operation to continue - cuts take place along the direction of the muscle fibres.

Once suitable access is achieved, the head of the femur is removed by a saw cut at the selected position. The incision must be large enough to allow access by the instrument and for the removal of the, by now superfluous, bone.

A reamer is used to prepare the interior of the femur to receive the prosthesis and the interior of the acetabulum to receive the cup that is to be inserted into the ileum.

The prosthesis is inserted into the stump of the femur. The placement should be solid, so planning where it will sit is an important aspect of the operation. The cup is inserted into the ileum and the prosthesis is fitted against it. Apart from a general restoration of tissue position and a closing of the skin incision, the operation is now complete.

It is essential that the position of the head of the prosthesis relative to the cup does not exert excessive strain on muscles and other tissue during subsequent movement of the patient. Thus, part of the planning of the operation relates to ensuring that the way in which the new joint moves is suitable to the anatomy of the patient.

\section{Creation of subject-specific model}

Due to the inclusion of soft tissue in the planning, the implemented pre-operative planner requires a complete anatomical model of the musculo-skeletal apparatus of the patient. In the optimal condition, CT and MRI of the patient hip joints are available, where CT data provide the skeletal anatomy and the outer body shape and MRI data the muscle and ligament anatomy. However in THR, standard clinical practice requires that only a $\mathrm{CT}$ scan of the patient is taken for planning purposes.

In order to provide a complete musculo-skeletal model of the patient, a pre-processing unit has been implemented in which a technician can scale a generic muscle anatomy atlas to fit the patient's skeletal anatomy. Thus, a musculo-skeletal model of the lower limb has been developed using the Visible Human dataset $(\mathrm{VH})$ [9]. From these data, the surfaces of all of the most important bones, muscles and tendons have been extracted to create a complete representation of the hip joint.

The pre-processing unit allows the $\mathrm{VH}$ model to be registered and scaled to the subject $\mathrm{CT}$ scan in order to complete a model of the subject's anatomy with all the relevant structures. In particular, the user can identify appropriate skeletal landmarks on the patient's bone surface. Then, the subject's muscle insertions and origins are estimated by an affine registration from the $\mathrm{VH}$ data. These data are used, together with the VH muscle surfaces, as input for the muscle surface registration environment in which the shape of each muscle can be properly adapted to the patient-specific anatomy (Fig. 1).

Further details of the Muscle Modelling Laboratory (MML) can be found in Krokos et al. [10].

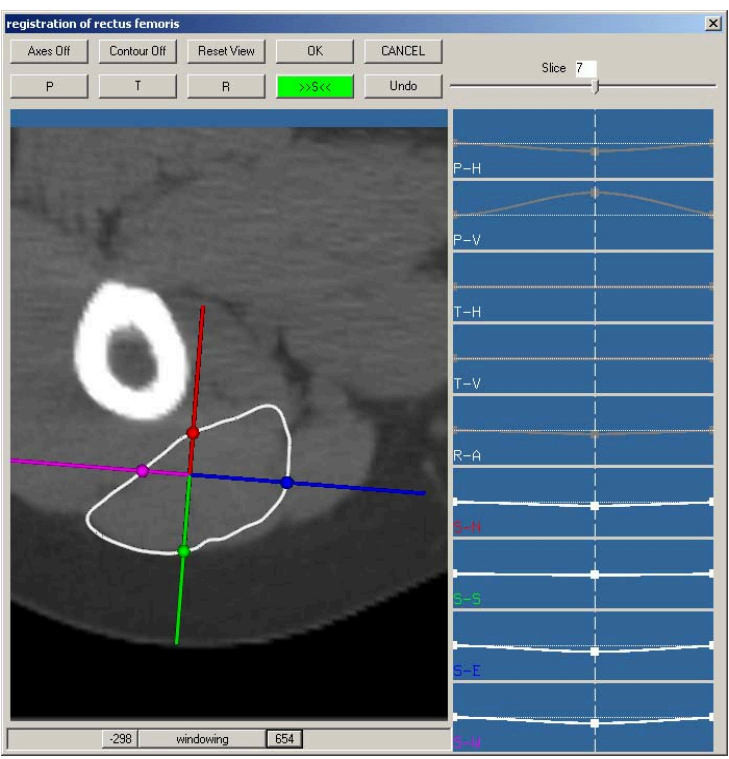

Figure 1. Muscle registration modules

\section{The pre-operative planning nvironment}

Once the data of the patient have been prepared and imported into the planning environment, the user can perform different actions and move between different software states. If desired, these transitions can be instigated by voice command.

\subsection{Surgical access planning}

In the first phase of the planning, the user estimates the surgical access. This modality is useful to ensure 
that a particular anatomical feature is visible/reachable during surgery, in particular when using minimally invasive approaches. The surgeon can visualise the patient's outer skin (and, using transparency, the muscles and the skeleton) and mark on the external surface the line of incision. At this point, the user can retract the skin to inspect the muscles behind (Fig. 2).

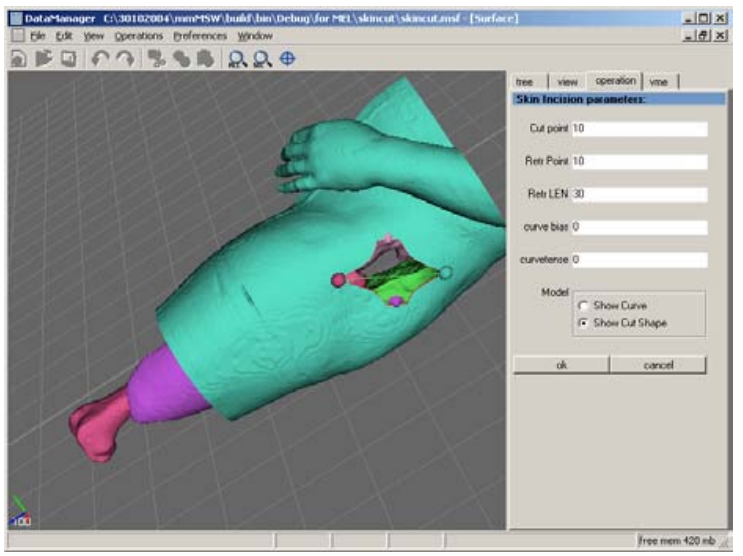

Figure 2. Skin retraction module

Then for each muscle, the surgeon can decide to cut or to retract. During the retraction, the muscle shape is deformed interactively and force feedback is given to the user in order to understand if there is muscle damage and if it is possible to retract the muscle sufficiently to provide access to the acetabular capsule (Fig. 3).

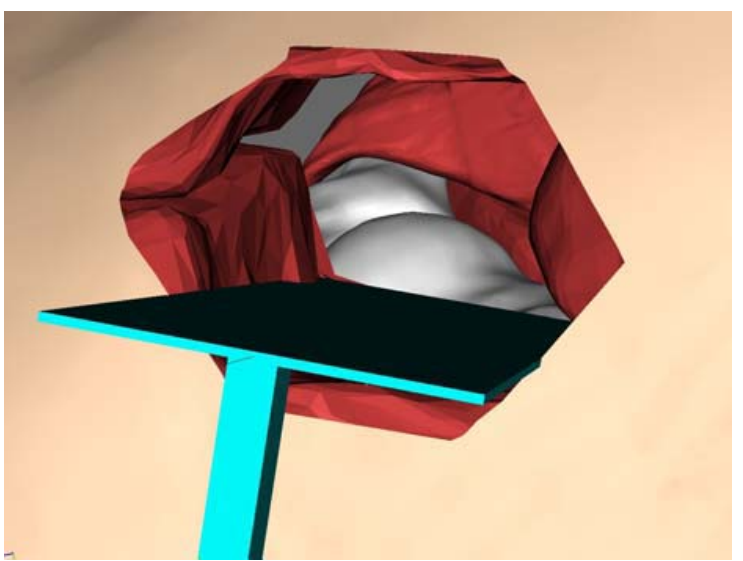

Figure 3. Muscle retraction module

At the end of the procedure (when the bones are visible within the access), the visualisation represents what the surgeon would see in the operating theatre.

\subsection{Prosthesis positioning planning}

Having selected a prosthetic component (both in type and size), the user moves to the prosthesis positioning phase. During this, a two-handed interaction is available: one hand is used to control the viewing position and the other to perform the sixdegrees-of-freedom positioning of the components. Naturally, during the course of a two-handed interaction, the availability of voice control is particularly useful.

At any time during the positioning, the surgeon can check a series of functional indicators: the feasibility of the planned position, the extension of bone-implant contact in cementless components, the primary stability of cementless components, the range of motion of the operated joint, the limb lengthening/shortening after joint reduction, the balancing of soft tissues, and the alteration of articular muscle moment arms (Fig. 4). The specific visualisation algorithms implemented are integrated with force feedback for some of the abovementioned biomechanical indicators, such as the range of motion, the feasibility of the planned position, and the primary stability evaluation.

Further details of the integration of haptics and visualisation in the context of this application is given in Mayoral et al. [11].

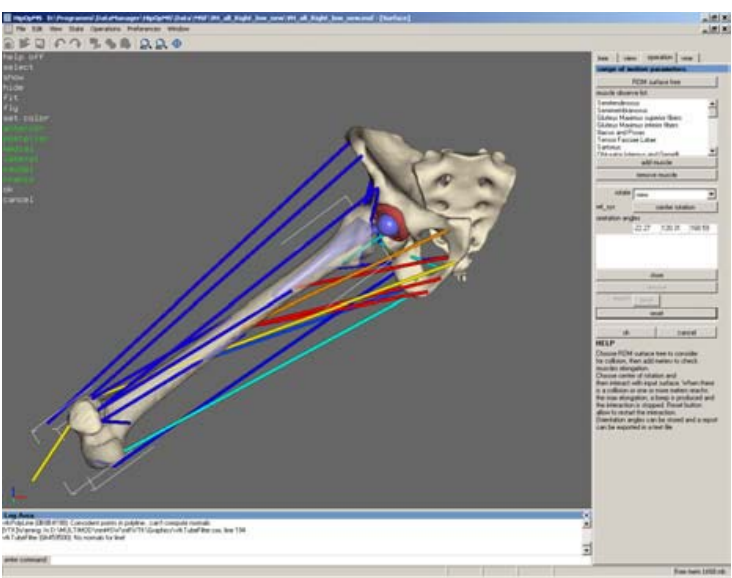

Figure 4. Range of motion evaluation module

\subsection{Surgical planning}

Once the pose of the prosthetic components is defined, the surgeon can ask the system to compute the neck resection plane as well as the insertion path of the reamer (Fig. 5). Once the surgical planning is accepted, the system generates a model of the postoperative anatomy on which the surgeon can perform final verification and inspection. 


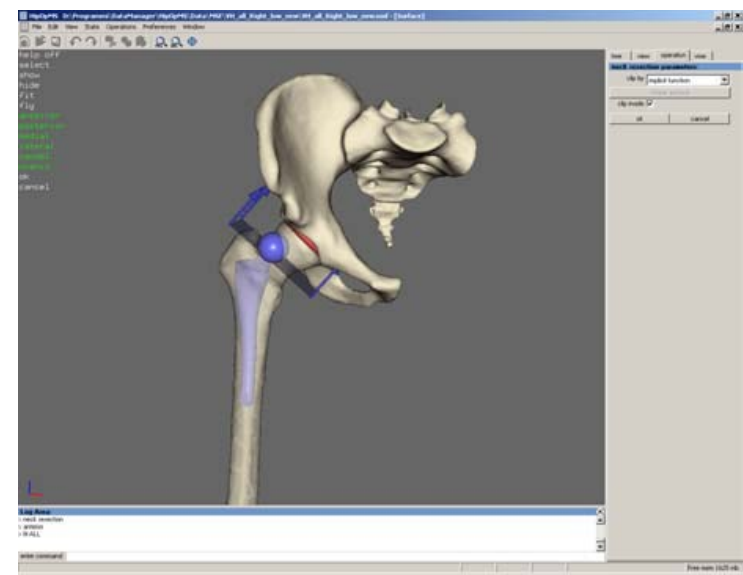

Figure 5. Neck resection module

\section{System implementation}

Developing a complex system, such as the one described here, requires a large number of basic algorithms and technologies to be integrated into single system. In recent years, many Open Source libraries and toolkits have been developed, providing a foundation for many application fields.

Well known toolkits, adopted in this work, are VTK [11] for visualisation, ITK [12] for segmentation and registration, and VXL [13] for numerical algorithms. Single libraries are at too low a level for the efficient development of a complex application, since this often implies that a base implementation has to be extended with new application modules. For this reason, several application frameworks have recently been developed.

Application frameworks have a sense of completeness, in that they are able to run by themselves, without any further work. One example is ParaView [14], used in fluid dynamics and general scientific visualisation problems. Another is the Julius framework [15], which was developed specifically for medical applications, but which provides also a limited number of visualisation modalities. Both are based on VTK, and Julius is also based on Qt library, which is not Open Source under Windows. Neither of these provides multimodal support.

The pre-operative planner for THR presented in this paper has been developed on top of the Multimod Application Framework (MAF) [16]. MAF is an Open Source application framework built on top of the VTK, ITK and wxWindows libraries and other Open Source and cross-platform software.

During the development of the current application, the MAF was extended by introducing support for multimodal interaction and provided with further visualisation algorithms (Fig. 6). The hardware implementation of the multimodal/ multisensorial system is presented in Fig. 6.

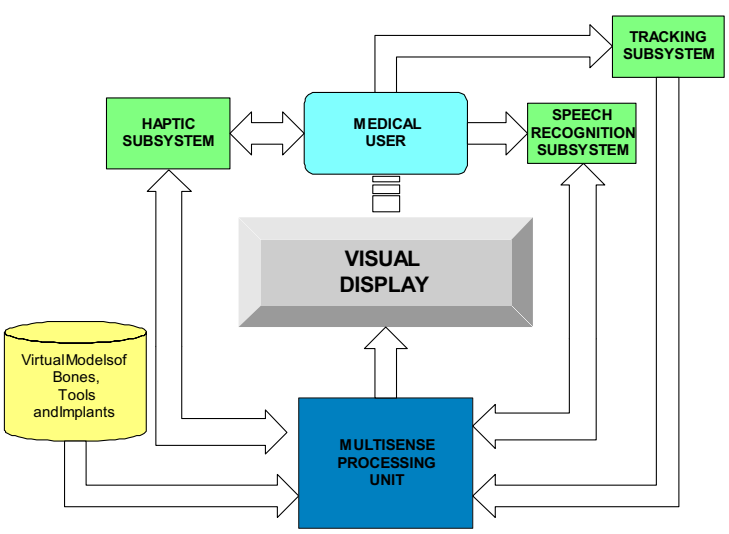

Figure 6. The multimodal system implementation

To address the intensive computational needs of the multimodal system and the different update rates required to control the different devices (the speech subsystem produces input at the rate of a few seconds, graphics modules at the rate of $10-30 \mathrm{msec}$, and haptic devices at the speed of less than a millisecond), the architecture is based on networked dedicated machines. In comparison to using a single machine, this multithreaded approach has some advantages: minimum coupling between local rates running on different machines, more consistent rates for critical processes such as the haptic servo input/feedback control, and provision of functionally separate, extensible and reconfigurable control of the different input feedback subsystems.

However, using multiple networked machines does increase the synchronisation requirements between the various subsystems implemented on the different machines. This has been directly addressed by the MAF. In our system, a dedicated graphics server machine is used to perform the graphics rendering; the low servo control and the input/feedback coordination of the haptic subsystem are managed by the haptic server machine; while a third server machine is responsible for the control of the speech subsystem. The communication between the various machines is performed using a TCP/IP protocol interface.

\section{Validation}

The application has been assessed with specific validation studies, using the following quantitative metrics: capabilities not provided by existing systems, inherent accuracy, time required to perform the task, global accuracy in performing the given task, intra- 
operator and inter-operators repeatability, and learning curve.

The different technologies integrated into the system have been independently validated with relevant but unambiguous tasks. The software modules providing quantitative indicators on the realised planning were also tested to assess the achievable accuracy and repeatability.

\section{Conclusion}

Pre-operative planning is a fundamental phase in total hip replacement. Helping the surgeon to acquire a more accurate mental model of the 3D relationships of the various tissues in the operational area for the specific patient can greatly increase the likelihood of a successful procedure.

However, the user interface in most surgical planning systems is mostly restricted to the use of a mouse and a flat (2D) screen. In this work, the application of multimodal/multisensorial interface technology in the pre-operative planning was presented. The interface was realised using advanced visualisation algorithms and with the integration of different interface units, including stereoscopic displays, haptics and speech. It is believed that true benefits can be gained from the use of this multimodal/multisensorial interface in all aspects of medical planning and training including the time required to perform a task, accuracy and repeatability in performing the task, and the learning curve.

\section{Acknowledgements}

This work was supported by the Multisense project (IST-2001-34121) of the Information Society Technologies Programme of the European Commission.

\section{References}

[1] H.D. Dobson, R.K. Pearl, C.P. Orsay, M. Rasmussen, R Evenhouse, Z. Ai, G. Blew, F. Dech, M.I. Edison, J.C. Silverstein and H. Abcarian, "Virtual reality: new method of teaching anorectal and pelvic floor anatomy", Dis Colon Rectum 2003;46(3):349-352.
[2] M.A. Spicer and M.L. Apuzzo, "Virtual reality surgery: neurosurgery and the contemporary landscape", Neurosurgery 2003;52(3):489-497; discussion 496-487.

[3] S. Hassfeld and J. Muhling, "Computer assisted oral and maxillofacial surgery--a review and an assessment of technology", Int J Oral Maxillofac Surg 2001;30(1):213.

[4] H. Handels, J. Ehrhardt, W. Plotz and S.J. Poppl, "Computer-assisted planning and simulation of hip operations using virtual three-dimensional models", Stud Health Technol Inform 1999;68:686-689.

[5] C.G. Schizas, B. Parker and P.-F. Leyvraz, "A study of pre-operative planning in CLS total hip arthroplasty", Hip International 1996;6:75-81.

[6] M. Viceconti, R. Lattanzi, B. Antonietti, S. Paderni, R. Olmi, A. Sudanese and A. Toni, "CT-based surgical planning software improves the accuracy of THR preoperative planning", Medical Engineering \& Physics $2003 ; 25(5): 371-377$

[7] S. Nishihara, N. Sugano, T. Nishii, H. Tanaka, H. Yoshikawa and A. Ochi, "Comparison of the fit and fill between the Anatomic Hip femoral component and the VerSys Taper femoral component using virtual implantation", J Orthop Sci 2003;8(3):352-360.

[8] B. Jaramaz, A.M.r. DiGioia, M. Blackwell and C. Nikou, "Computer assisted measurement of cup placement in total hip replacement", Clin Orthop 1998;354:70-81.

[9] National Library of Medicine, Visible Human project, http://www.nlm.nih.gov/research/visible/visible human.htm 1

[10] M. Krokos, D. Podgorelec, G.J. Clapworthy, D. Testi and M. Viceconti, "Patient-Specific Muscle Models for Surgical Planning”, Proc. MediViz 05, 2005

[11] R. Mayoral, N.G. Tsagarakis, G.J. Clapworthy, R. Liang, D.G. Caldwell, "Integration of Haptic and Visual Modalities in a Total Hip Replacement Planning System”, Proc. MediViz 05, 2005

[12] Visualization Tool Kit, Kitware Inc, http://www.vtk.org

[13] Insight Tool Kit, Kitware Inc., http://www.itk.org

[14] VXL collection of $\mathrm{C}++$ libraries, The TargetJr Consortium, http://paine.wiau.man.ac.uk/pub/doc vxl/books/core/book.html

[15] Parallel Visualization Application (ParaView), Kitware Inc., http://www.paraview.org

[16] Julius Software Framework, Surgical Systems Lab, http://www.julius.caesar.de

[17] M Viceconti et al., "The Multimod Application Framework", Proc. IEEE Conf. Information Visualisation, IV-04, pp 15-20, 2004 